\title{
The Role of Stratification-Dependent Mixing for the Stability of the Atlantic Overturning in a Global Climate Model*
}

\author{
BEn MARZEION \\ Nansen Environmental and Remote Sensing Center, and Bjerknes Centre for Climate Research, Bergen, Norway \\ ANDERS LEVERMANN \\ Earth System Analysis, Potsdam Institute for Climate Impact Research, and Institute of Physics, Potsdam University, \\ Potsdam, Germany \\ Juliette Mignot \\ LOCEAN, Université Pierre et Marie Curie, Paris, France
}

(Manuscript received 8 June 2006, in final form 31 January 2007)

\begin{abstract}
Using the "CLIMBER-3 $\alpha$ " coupled climate model of intermediate complexity, the effect of a stratification-dependent vertical diffusivity on the sensitivity of the Atlantic Ocean meridional overturning circulation to perturbations in freshwater forcing is investigated. The vertical diffusivity $\kappa$ is calculated as $\kappa \sim N^{-\alpha}$, where $N$ is the local buoyancy frequency and the parameter $\alpha$ is a measure of the sensitivity of the vertical diffusivity to changes in stratification. Independent of $\alpha$, the stratification of the deep ocean is weakly increased as a response to an anomalous freshwater flux in the North Atlantic in these experiments. In the region of freshwater forcing and north of it this is caused by the freshwater anomaly itself, but farther south it is associated with anomalously warm surface waters caused by a reduction of the northward oceanic heat transport. Subsequently, and in opposition to results from previous studies, the overturning is reduced by the anomalous freshwater flux, independent of the choice of $\alpha$. However, the amount of reduction in overturning following a freshwater perturbation is found to depend critically on the choice of the mixing sensitivity $\alpha$. If $\alpha<\alpha_{\text {cr }}$, the response is similar to the model's response using constant vertical diffusivity $(\alpha=0)$. For $\alpha>\alpha_{\mathrm{cr}}$, a sharp increase of the sensitivity is found. The value of $\alpha_{\mathrm{cr}}$ is found to be between 0.5 and 1. A general feedback is proposed explaining this threshold behavior: if $\alpha$ is large, both positive and negative perturbations of stratification are amplified by associated changes in diffusivity. In the experiments presented here, this enhances the initial positive stratification anomaly in northern high latitudes, which is created by the anomalous freshwater flux. As a result, convection is strongly reduced, and the overturning is significantly weakened.
\end{abstract}

\section{Introduction}

Past abrupt changes in the North Atlantic Ocean surface temperatures, as seen, for example, in temperature reconstructions from Greenland ice cores, are often explained by changes in the oceanic northward heat trans-

\footnotetext{
* Bjerknes Centre for Climate Research Publication Number A160.

Corresponding author address: Ben Marzeion, Nansen Environmental and Remote Sensing Center, Thormøhlensgate 47, 5006 Bergen, Norway.

E-mail: ben.marzeion@nersc.no
}

port (Clarke et al. 2002; Rahmstorf 2002). Those fluctuations could be caused by changes of the strength of the Atlantic meridional overturning circulation (AMOC), possibly triggered by variability in freshwater forcing of the North Atlantic Ocean (Ganopolski and Rahmstorf 2001; Vellinga et al. 2002). The prospect of a strengthened hydrological cycle as a response to greenhouse gas forcing and a likely increase of the meltwater runoff from Greenland (Church et al. 2001) have further lead to an interest in experiments that artificially slow down or collapse the Atlantic meridional overturning circulation by increasing the freshwater flux to the North Atlantic Ocean (e.g., Stocker and Wright 1991; Manabe and Stouffer 1995; Rahmstorf 1996; Rind et al. 2001; Rahmstorf et al. 2005; Stouffer et

DOI: $10.1175 / 2007 J P O 3641.1$

(C) 2007 American Meteorological Society 
al. 2006). In these so-called freshwater hosing experiments, the reduced surface salinity increases the stratification in high northern latitudes, which leads to less dense water being formed, and to a reduced meridional density gradient, which reduces the overturning.

Theoretical considerations (Munk and Wunsch 1998; Huang 1999) and model experiments (e.g., Marotzke and Scott 1999) indicate that vertical mixing is limiting the strength of the AMOC on long time scales [for a review. see Kuhlbrodt et al. (2007)]. At the same time, vertical diffusion is thought to be one of the processes influencing the strength of the AMOC that is least understood, and is inadequately represented in current ocean general circulation models (Zickfeld et al. 2007). It is therefore important to gain insight both into what is setting the strength of vertical diffusion in the ocean, how this influences the ocean circulation, and which implications this might have for interpreting model results.

In ocean general circulation models, vertical diffusivity is often set constant in time and/or in space. Hasumi and Suginohara (1999) developed a parameterization for enhanced mixing over rough topography, and the $K$-profile parameterization (Large et al. 1994) is often employed to improve the representation of the mixed layer. However, the vertical diffusivity of the entire ocean interior is influenced by stratification, among other things. That is because more turbulent kinetic energy is required to displace water across a strong vertical density gradient (Gargett and Holloway 1984). For modeling studies looking at small perturbations of the system around its equilibrium state, vertical diffusivity that is constant in time but varying with depth can be taken into account by using a typical profile of stratification. However, this approach fails as soon as major changes to the deep ocean water mass properties are involved.

One approach is to write the diffusivity as $\kappa \sim N^{-\alpha}$, where $\alpha$ is a parameter and

$$
N=\left(-\frac{g}{\rho_{0}} \frac{\partial \rho}{\partial z}\right)^{1 / 2}
$$

is the local buoyancy frequency, with $g$ being the gravity acceleration, $\rho_{0}$ being a reference density, and $\partial \rho / \partial z$ being the vertical density gradient. Gregg (1989) and Gargett and Holloway (1984) suggest the relation between the rate of dissipation of turbulent kinetic energy per mass $\varepsilon$ and $N$ to be $\varepsilon \sim N^{m}$, with $1 \leq m \leq 2$. Using $\kappa \sim \varepsilon / N^{2}$, this corresponds to $0 \leq \alpha \leq 1$. Studies using vertical profiles of the tracers ${ }^{222} \mathrm{Rn}$ and ${ }^{228} \mathrm{Rn}$ (Sarmiento et al. 1976) and bomb-produced tritium (Hoffert and Broecker 1978; Broecker and Peng 1982) find that $\alpha \approx 2$, corresponding to a constant energy dissipation rate. Rehmann and Duda (2000) observed that $\alpha=3.1$ close to the bottom of a shelf slope. While the diversity of these numbers illustrates that obviously stratification is not the only variable setting the vertical diffusivity, it also points out that the assumption of a constant diffusivity is poorly justified.

Nilsson and Walin (2001) use a two-layer model of the Northern Hemisphere Atlantic to investigate the cases $\alpha=0,1$, and 2 . They identify two regimes in the model: a freshwater-impeded regime for $\alpha=0$, which corresponds to constant vertical diffusivity, and a freshwater-boosted regime for $\alpha=1$ and 2, in which the $\mathrm{MOC}$ is increasing as a response to stronger freshwater forcing of the North Atlantic. This response is caused by the freshwater anomaly getting advected southward with the deep return flow, causing the stratification to weaken, and leading to an increase of the diapycnal diffusivity, which is finally causing the rate of overturning to increase. Using an idealized setup for an OGCM with only one active tracer (temperature), Nilsson et al. (2003) find a very good match between model results and theoretical considerations based on scaling laws derived in Nilsson and Walin (2001). Marzeion and Drange (2006) extended this study using a conceptual oceanic model based on the model proposed by Gnanadesikan (1999), similar to the one by Nilsson and Walin (2001) and Oliver et al. (2005). They identify the critical value of $\alpha$, at which the freshwater-impeded regime changes into the freshwater-boosted regime, to be $\alpha \approx 0.75$.

In this study, we use a coupled climate model of intermediate complexity to investigate the effect of different values of $\alpha$ on the sensitivity of the AMOC. As opposed to the idealized model employed by Nilsson et al. (2003), our model system captures the effects of both temperature and salinity on changes in density and resolves the effect of different boundary conditions for those two active tracers. Also, our model is global so that possible effects from the Southern Ocean are included. The aim of this study is to identify the processes found in previous studies in the coupled model employed in this study. The model is presented in the next section together with the experimental setup. The results of previous studies were based on changes of the stratification and diffusivity in the upwelling branch of the overturning in low latitudes. In section 3, we present the effect that the parameterization has on the diffusivity in low latitudes in the "CLIMBER-3 $\alpha$ " model. In section 4, we suggest a new feedback working on the downwelling branch of the overturning in the high northern latitudes, causing a critical increase of the sensitivity of the overturning to freshwater. In section 5, 
we discuss why the freshwater-boosted regime, as found by Nilsson and Walin (2001) and others, is not found in our model, and explain the possible implications of our findings for understanding the AMOC system and for future research. Our results are summarized in section 6 .

\section{Numerical experiments}

\section{a. Model description}

In this study we use the CLIMBER-3 $\alpha$ earth system model of intermediate complexity (Montoya et al. 2005). It combines an ocean general circulation model based on the Geophysical Fluid Dynamics Laboratory Modular Ocean Model, version 3, (MOM-3) code, the Potsdam Statistical-Dynamical Atmospheric Model (POTSDAM-2; Petoukhov et al. 2000), and the dynamic and thermodynamic sea ice module of Fichefet and Maqueda (1997). The oceanic horizontal resolution is $3.75^{\circ} \times 3.75^{\circ}$, and there are 24 vertical levels. The applied advection-diffusion scheme (Prather 1986) minimizes numerical diffusion (Hofmann and Maqueda 2006). For a study of the sensitivity of the model to constant vertical diffusivity see Mignot et al. (2006).

The atmospheric component has a resolution of $7.5^{\circ}$ latitude and $22.5^{\circ}$ longitude and is assuming a universal vertical structure of temperature and humidity, reducing the three-dimensional description to two-dimensional prognostic equations for temperature and humidity. Heat and freshwater fluxes between ocean and atmosphere are computed on the ocean grid, with no flux adjustment being employed. Since the atmospheric grid is too coarse to provide realistic wind stress forcing, in this study climatological National Centers for Environmental Prediction-National Center for Atmospheric Research reanalysis data are used for the momentum flux.

\section{b. Parameterization of vertical diffusivity}

The background vertical diffusivity $\kappa$ was calculated depending on the local buoyancy frequency $N$ as

$$
\kappa=\kappa_{0}\left(\frac{N}{N_{0}}\right)^{-\alpha}
$$

where $N_{0}$ is chosen to represent a typical value of $N$ around pycnocline depth, so that the diffusivity in the pycnocline is primarily controlled by the choice of $\kappa_{0}$. The parameter $\alpha$ is the normalized derivative of $\kappa$ at $N=N_{0}$,

$$
\alpha=-\left.\frac{\partial\left(\kappa / \kappa_{0}\right)}{\partial\left(N / N_{0}\right)}\right|_{N=N_{0}},
$$

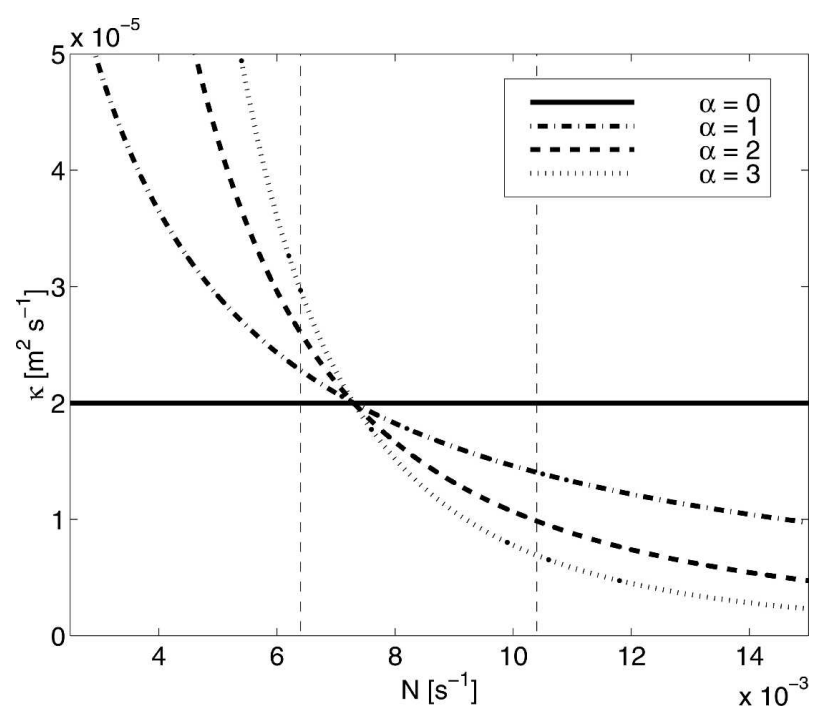

FIG. 1. Vertical diffusivity $\kappa$ as a function of buoyancy frequency $N$ for the standard set of parameters used in the experiments. The vertical dashed lines indicate the range of $N$ found in the unperturbed experiments around pycnocline depth (i.e., between 450 and $800 \mathrm{~m})$.

and is thus controlling the sensitivity of the vertical diffusivity to changes in stratification. The aim of this parameterization is to allow for changes in $\alpha$, without changing the diffusivity at pycnocline depth of the unperturbed ocean. Thus it is possible to attribute changes in the sensitivity of the MOC to different values of $\alpha$, instead of different equilibrium ocean states. To better represent mixed layer processes, the $K$-profile parameterization (Large et al. 1994) is employed in the upper layer.

Figure 1 shows $\kappa$ as a function of $N$, using $N_{0}=7.3 \times$ $10^{-3} \mathrm{~s}^{-1}$, and $\kappa_{0}=0.2 \times 10^{-4} \mathrm{~m}^{2} \mathrm{~s}^{-1}$. Depending on the value of $\alpha$, this parameterization results in $0.05 \times 10^{-4}$ $\mathrm{m}^{2} \mathrm{~s}^{-1} \lesssim \kappa \lesssim 0.3 \times 10^{-4} \mathrm{~m}^{2} \mathrm{~s}^{-1}$ in the global ocean around pycnocline depth in the equilibrium runs. In the deep ocean, where the stratification is weak, values of $\kappa$ exceed $1.5 \times 10^{-4} \mathrm{~m}^{2} \mathrm{~s}^{-1}$ for $\alpha=3$.

Figure 2 shows the time series of the maximum of the Atlantic meridional overturning streamfunction for the model runs discussed in this study. Mignot et al. (2006) showed that the rate of overturning increases quasilinearly with the vertical mixing coefficient in the pycnocline. Depending on the value of $\alpha$, the overturning strength of the initial equilibrium in our runs is about $12-13 \mathrm{~Sv}\left(1 \mathrm{~Sv} \equiv 10^{6} \mathrm{~m}^{3} \mathrm{~s}^{-1}\right)$, and the strength generally decreases with increasing $\alpha$. This indicates that the value of $N_{0}$ was chosen slightly too low to generate the same vertical mixing in the pycnocline for all values of $\alpha$. However, the model states are actually very close to each other considering the variations that have been 

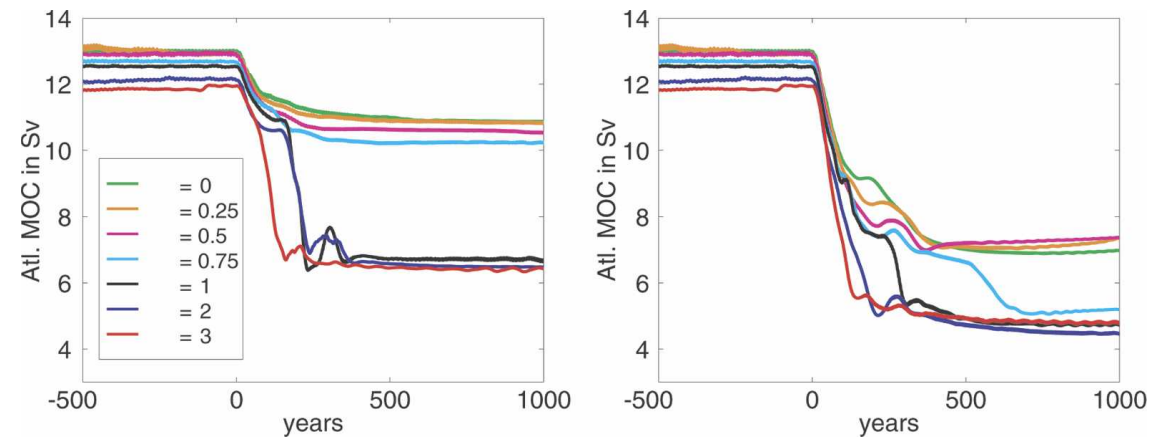

FIG. 2. Time series of the maximum of the Atlantic meridional overturning, filtered with a 20 -yr running mean. The freshwater flux starts at $t=0 \mathrm{yr}$. The (left) sflx01 runs and (right) sflx02 runs.

introduced into the diffusivity, especially for high values of $\alpha$ (see Fig. 1).

\section{c. Freshwater experiments}

First, the model is run to equilibrium with unperturbed forcing for about $2000 \mathrm{yr}$. Then a freshwater flux anomaly is simulated by imposing a virtual negative salt flux between $20^{\circ}$ and $50^{\circ} \mathrm{N}$ in the Atlantic, with two sets of experiments corresponding to $0.1 \mathrm{~Sv}$ (sflx01 experiments) and $0.2 \mathrm{~Sv}$ (sflx02 experiments), respectively. The model is then run for at least another $1000 \mathrm{yr}$, until the strength of the Atlantic MOC has reached a new equilibrium.

Since the virtual salt fluxes were used to mimic an increased freshwater input from external sources, such as glacier runoff, they were not compensated for in the model runs presented here. However, we tested the sensitivity of our results to compensation of the salt fluxes in a large domain of the Pacific Ocean. While this prevents a drift in the global salinity, it did not change the dynamics described in this paper. Furthermore, a number of runs were repeated using the sum of climatological wind stress and the wind stress anomaly calculated by the atmospheric model relative to the control runs (for a detailed description see Montoya et al. 2005). While this procedure ensures that effects of changes in the wind stress are included in the model response, no significant deviations from the runs presented in this study are found.

\section{Response at low latitudes}

When the anomalous freshwater flux is started, the overturning weakens for all values of $\alpha$ (see Fig. 2), as opposed to results from previous studies using the same parameterization of vertical diffusivity (Nilsson and Walin 2001; Marzeion and Drange 2006). This result is similar to studies using models employing a parameterization corresponding to $\alpha=0$ (see, e.g., Stouffer et al. 2006): the stabilization of the water column in high latitudes caused by the anomalous freshwater flux leads to a weakening of the AMOC. North of about $10^{\circ} \mathrm{N}$ the freshwater causes a negative salinity anomaly that is first advected northward, penetrates the deep ocean there, and is then advected southward with the deep return flow of the overturning. Figure 3 illustrates the temporal evolution of the zonally averaged salinity and temperature anomalies after the anomalous freshwater flux is started. While the freshwater is creating a negative salinity anomaly within and north of the freshwater forcing region (black contours), a warm anomaly is building up in the surface south of the forcing region (red contours). This is caused by a reduction of the northward heat transport as seen in Fig. 4. Since the reduction in the sflx02 case is slightly stronger than in the sflx 01 case, the heat anomaly is stronger in the sflx02 case.

Figure 5 shows the resulting changes in the zonally averaged density. The light anomaly created by the anomalous freshwater flux stretches across the whole water column above $\sim 2000 \mathrm{~m}$. At low latitudes, the light anomaly of the upper layers is due to the warm anomaly rather than the direct effect of the freshwater. This thermal effect was not seen in the experiments by Nilsson and Walin (2001) and Marzeion and Drange (2006) and causes the stratification at pycnocline depth and in the deep ocean at low latitudes to increase, if only weakly. The depth of the pycnocline thus proves to be very stable, as already shown by Levermann and Griesel (2004) for constant vertical diffusivity. In conclusion, the mechanism that was proposed by Nilsson and Walin (2001) and also found by, for example, Marzeion and Drange (2006), is not at play in this model. 

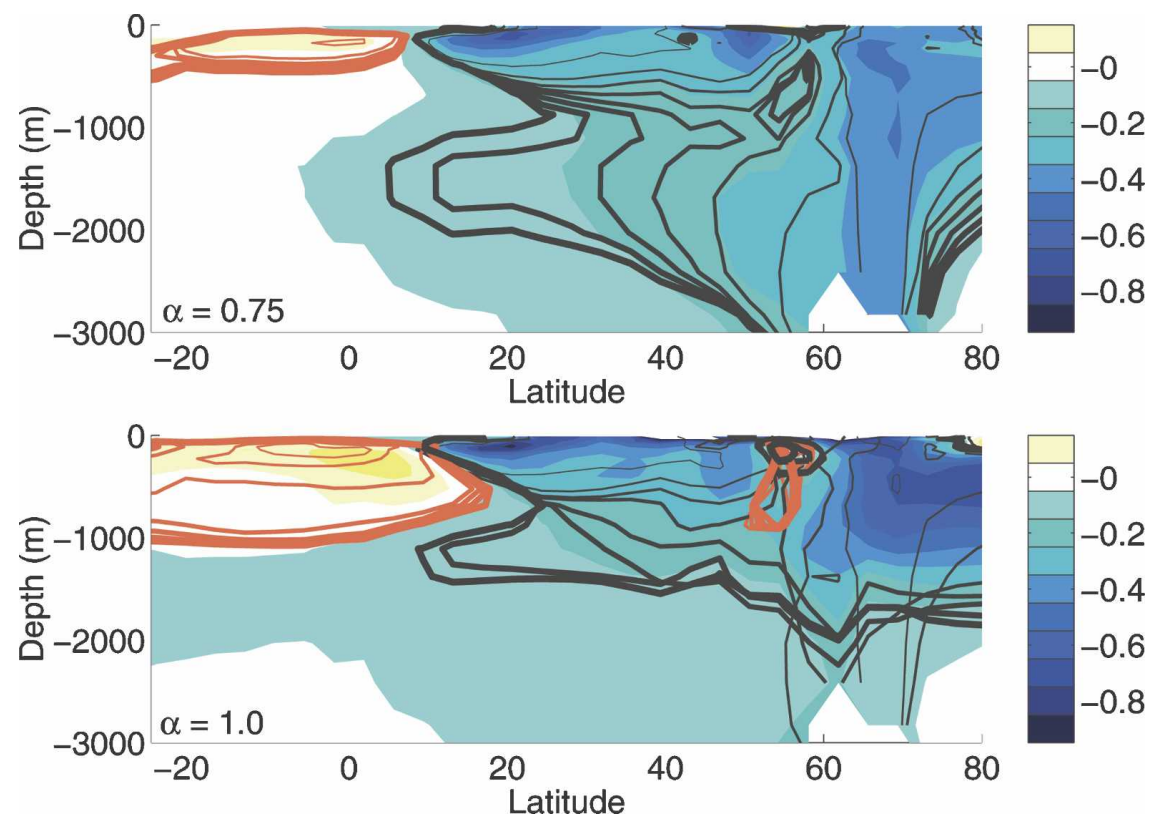

FIG. 3. Temporal evolution of the temperature and salinity anomalies: (top) $\alpha=0.75$ and (bottom) $\alpha=1$. Background shading: salinity anomaly (psu) after $300 \mathrm{yr}$ of $0.1-\mathrm{Sv}$ anomalous freshwater flux. Black lines: position of the zonally averaged -0.2 -psu salinity anomaly, starting at $t=100 \mathrm{yr}$ with the thinnest line; line thickness increasing with 50 -yr time steps. Last (i.e., thickest) line shows the position at $t=450 \mathrm{yr}$. Red lines: the same as black, but for the zonally averaged $0.7-\mathrm{K}$ temperature anomaly. The values of the contours were chosen such that they represent approximately the same change in density.

\section{The response at high latitudes}

While the response of the overturning is similar for all values of $\alpha$ at low latitudes, the response at high latitudes depends critically on the value of $\alpha$. Figure 2 shows that for each set of experiments, the runs can be classified in two groups, separated by a critical value of $\alpha=\alpha_{\text {cr. }}$. In the sflx01 case, $0.75<\alpha_{\text {cr }}<1$ : For all the runs with $\alpha<\alpha_{\text {cr }}$ the overturning is reduced by $\sim 2 \mathrm{~Sv}$ (subcritical runs). For all runs with $\alpha>\alpha_{\mathrm{cr}}$, the reduction is significantly larger around $5.5 \mathrm{~Sv}$ (supercritical runs). In the sflx02 experiments $0.5<\alpha_{\text {cr }}<0.75$, and the overturning is reduced by 6 and $8 \mathrm{~Sv}$ for the subcritical and supercritical runs, respectively. Note that the sflx02 experiment using $\alpha=0.75$ switches between the two groups only after $500 \mathrm{yr}$ of anomalous freshwater flux.

The difference between these two groups originates from the part of the overturning lying north of $65^{\circ} \mathrm{N}$ : In the supercritical runs, the overturning streamfunction is reduced to 0 north of the ridges as opposed to the subcritical run's behavior (see Fig. 5). The response of the
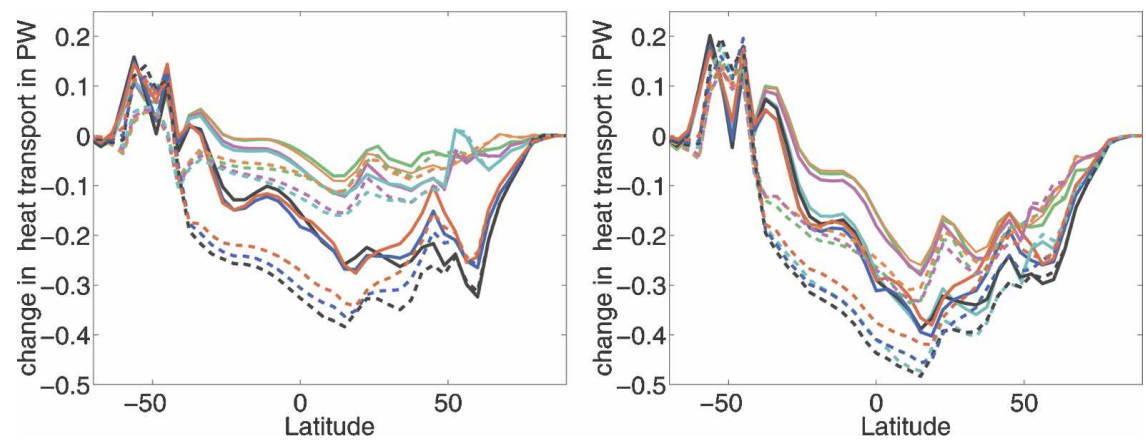

FIG. 4. Change in global (solid lines) and Atlantic (dashed lines) oceanic northward heat transport after $800 \mathrm{yr}$ of (left) 0.1- and (right) 0.2-Sv freshwater flux. Colors are as in Fig. 2. 

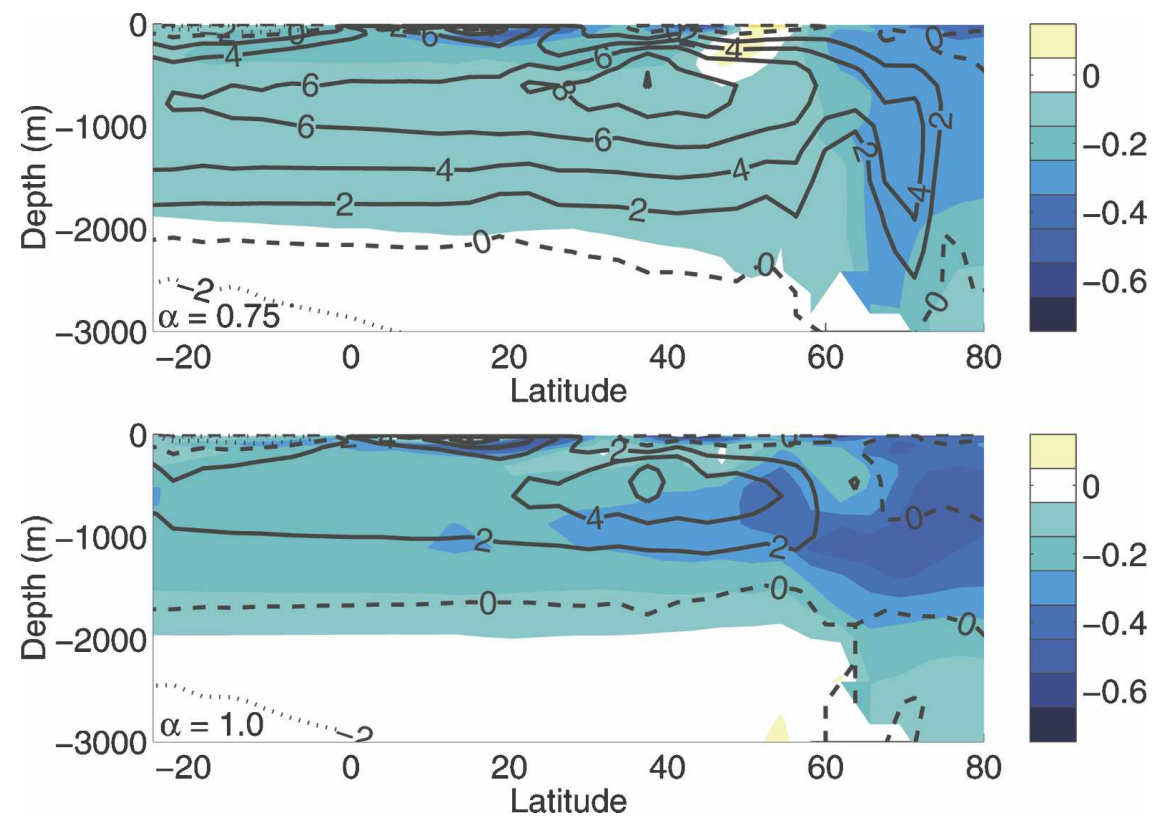

FIG. 5. Shading: zonally averaged density anomalies $\left(\mathrm{kg} \mathrm{m}^{-3}\right)$ after $800 \mathrm{yr}$ of $0.1-\mathrm{Sv}$ freshwater flux. Contours: overturning streamfunction (Sv) at the same time. (top) $\alpha=0.75$ and (bottom) $\alpha=1$.

overturning at lower latitudes however remains dependent on the rate of the anomalous freshwater flux as described in section 3. This indicates that the global response of the overturning streamfunction can be understood as a superposition of a weak reduction of the overturning streamfunction that is present in all runs, and a strong, nonlinear reduction of the overturning streamfunction that originates in the Nordic seas and is only present in the supercritical cases.

It is helpful to discuss a highly idealized two-layer model of dense-water formation to understand this nonlinear behavior: during the summer months, a positive buoyancy flux from the atmosphere creates a buoyancy reservoir in the upper layer; during the winter months, a negative buoyancy flux decreases the amount of buoyancy stored in the reservoir. At the same time, diffusion between the upper and lower layers decreases the amount of buoyancy of the upper layer by increasing the density of the upper layer and decreasing that of the lower layer. In this simplified model, dense-water formation occurs when the water of the upper layer gets denser than the water of the lower layer. This means that dense-water formation occurs if the negative buoyancy flux integrated over one winter season is greater then the amount of buoyancy stored in the upper layer relative to the lower layer.

In the equilibrium runs, dense-water formation is occurring for all values of $\alpha$. This indicates that independent of the diffusion between upper and lower layers, the amount of buoyancy stored in the upper layer is always smaller than the integrated buoyancy flux during the winter season. By adding freshwater, the amount of buoyancy in the upper ocean layer is increased. At the same time, following Eq. (1), the diffusion between the two layers is reduced. The reduction in diffusion increases with $\alpha$, so that the increase of buoyancy in the upper layer increases with $\alpha$. Thus, depending on $\alpha$ and on the amount of freshwater added to the upper layer, the buoyancy flux during the wintertime may not be enough to empty the reservoir of buoyancy, so that dense-water formation does not occur. Once this happens, part of the buoyancy reservoir is carried over into the next season. Since this makes deep-water formation even less likely in the following years, the mechanism has the characteristics of a oneway (i.e., off) switch. The value of the critical $\alpha$ should decrease with increasing freshwater forcing, as we observe it in our experiments.

This mechanism can be observed in our model results: Fig. 3 shows that both for subcritical (top) and supercritical (bottom) runs, the freshwater initially penetrates to depth in the Nordic seas, indicating that dense-water formation is happening. However, as described above, the freshwater flux increases the stratification in the areas of dense-water formation. While the freshwater anomaly continues to be evenly distributed in the water column in the subcritical case, it gets confined to the upper $1500 \mathrm{~m}$ of the water column after 


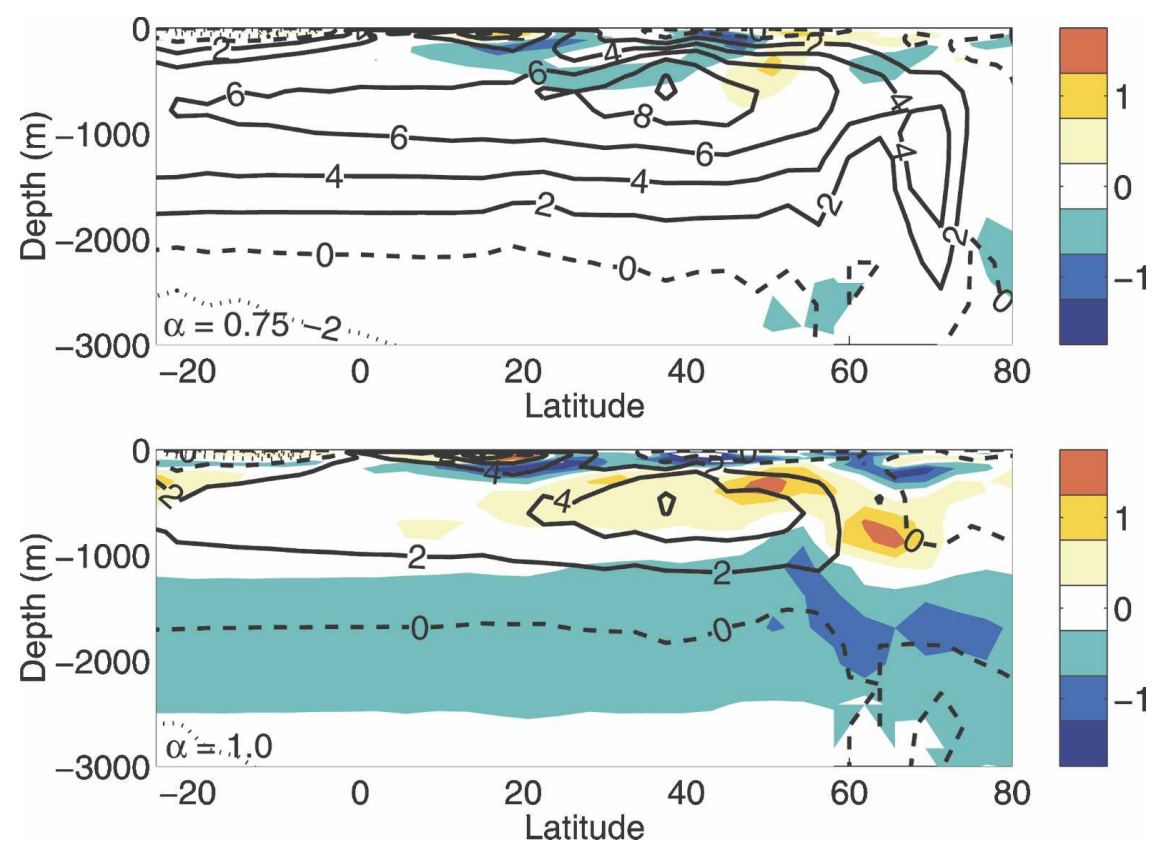

FIG. 6. Same as in Fig. 5, but shading denotes zonally averaged anomalies of the background diffusivity $\kappa\left(10^{-6} \mathrm{~m}^{2} \mathrm{~s}^{-1}\right)$.

$\sim 200 \mathrm{yr}$ in the supercritical case. At that time, deepwater formation ceases, and the overturning is further reduced. In the supercritical case, Fig. 6 shows a decrease of diffusivity around $1500-\mathrm{m}$ depth that is weak in the low latitudes, but increases north of $65^{\circ} \mathrm{N}$. It is not present in the subcritical case. In Fig. 5 a strong light anomaly can be seen in the upper $\sim 1500 \mathrm{~m}$, corresponding to a large amount of buoyancy. The mixed layer depths plotted in Fig. 7 confirm that the anomalous freshwater flux only has a weak influence on deep convection in the Nordic seas in the subcritical case, while deep convection completely ceases in the supercritical case.

\section{Discussion}

Nilsson et al. (2003) show evidence of an increase of the overturning for high values of $\alpha$ using a general circulation model, in very close accordance to the results predicted from conceptual models (Nilsson and Walin 2001; Marzeion and Drange 2006), which were derived from advective-diffusive scaling. But the results presented in this study contradict the predictions obtained from the simplified models. Specifically, increasing the freshwater flux into the North Atlantic Ocean did not result in a weakened stratification. There are three differences between the model used by Nilsson et al. (2003) and the model used in this study that one could invoke to explain this behavior:
1) A major difference of the model applied in this study from the previous numerical study by Nilsson et al. (2003) is the inclusion of the global ocean. As pointed out by Scott et al. (1999) and Klinger and Marotzke (1999), for an interhemispheric overturning circulation the ratio between southern and northern ocean freshwater fluxes is crucial for determining the stability of the MOC. The implications of applying an asymmetric freshwater flux can therefore not be predicted from a hemispheric ocean model.

In CLIMBER-3 $\alpha$, Antarctic Bottom Water $(\mathrm{AABW})$ is formed in the realm of Antarctica and causes a reversed overturning cell in the lowest layers of the Atlantic Ocean. As the AMOC is weakened by the anomalous freshwater flux, the AABW cell expands vertically, but it does not increase in strength. The expansion of the AABW cell corresponds to a decrease in the vertical extension of the AMOC. Without taking changes in density into account, this implies a compression of the vertical axis in the AMOC, contributing to the increase in stratification.

Also, Marotzke and Klinger (2000) found that the magnitude of the interhemispheric transport depends strongly on the diapycnal mixing in each of the hemispheres. As a consequence, discussing the effects of stratification-dependent mixing in only one hemisphere may be insufficient to predict the behavior of a cross-equatorial overturning. 

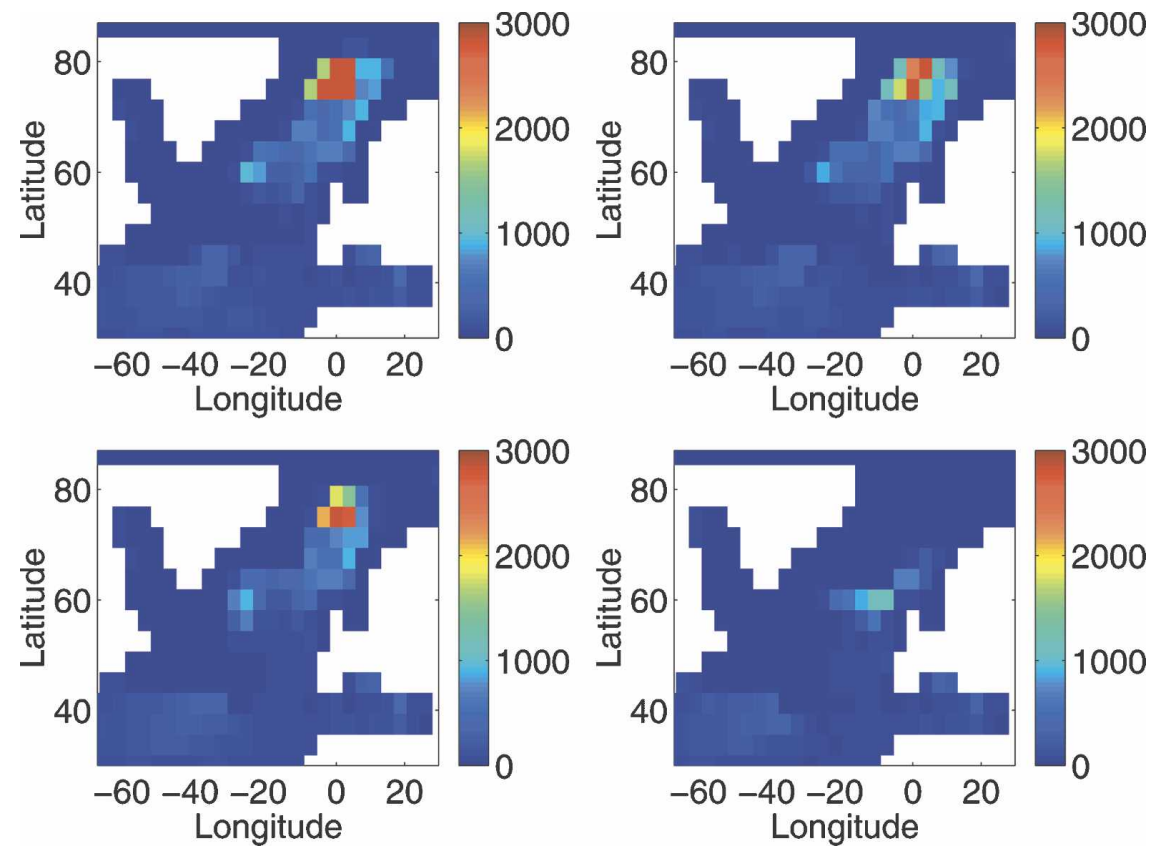

FIG. 7. Annual maximum of the mixed layer depths: (left) $\alpha=0.75$ and (right) $\alpha=1$. (top) Unperturbed runs and (bottom) after $800 \mathrm{yr}$ of 0.1-Sv freshwater flux.

2) We did not address the role of the atmospheric coupling for our findings. We believe that the main effects found in our experiments do not depend critically on ocean-atmosphere feedbacks but wish to point out that in an uncoupled model, such as the one used by Nilsson et al. (2003), the changes in low-latitude diffusivity might be stronger, further decreasing the strength of the AMOC by weakening the upwelling branch. Figure 3 shows that the regions of freshening and warming do not overlap, but rather form a common envelope. Very close to the surface, the warm anomaly vanishes. This may indicate that the freshwater anomaly is recirculating southward in the subtropical gyre, where increased evaporation caused by the warm anomaly erases the fresh signal, and cools the surface ocean. Future studies are necessary to address this possible damping effect of the atmosphere.

3) Nilsson et al. (2003) use a model that is driven by temperature gradients only. Therefore, the reduction of the surface meridional density gradient was achieved by weakening the amplitude of the temperature profile that was used to force the ocean at the surface. Here, the reduction of the surface meridional temperature gradient was achieved by increasing the freshwater flux into the North Atlantic Ocean. Comparing these two cases directly is problematic.
Consider a circulation along closed streamlines that is exposed to a temperature gradient forcing along the surface. In the limit of weak diffusion, a water parcel will not change its density while drifting at depth from the high to the low latitudes. If the temperature gradient at the surface is now changed, the water parcels will carry the changed surface temperatures to depth, and at equilibrium, all temperature gradients along one streamline away from the surface vanish. However, if an external freshwater source is added somewhere along the circulation path, this will result in a mean salinity drift of the system. This drift is communicated into the deep ocean along the advection pathway, and salinity differences may exist between two water parcels that are located at different positions along the same streamline while the circulation itself is in equilibrium. This means that in the study presented here, the assumption that in equilibrium the properties of the water in the deep southward return flow of the MOC are the same as those of the surface water in the areas of dense-water formation may not be justified.

However, a few experiments were repeated with a compensation of the freshwater flux over a large area in the Pacific Ocean. This compensation had only very minor effects on the response of the MOC to the increased freshwater flux, which indicates that 
this difference to Nilsson et al. (2003) cannot explain our results.

Our results indicate that there may exist a feedback between stratification and vertical mixing that has not been described before and that may be powerful enough to substantially increase the sensitivity of the AMOC to freshwater forcing. Even though our results seem to contradict results from previous studies, they can be understood as different realizations of the same feedback. In the experiments presented here, it is the amplification of a positive stratification anomaly in high latitudes that is causing the strongly nonlinear response of the system. In previous studies, it was a similar positive feedback amplifying a negative anomaly of the stratification in low latitudes.

The halt of dense-water formation is occurring in the Nordic seas, which are separated from the North Atlantic by the Greenland-Scotland Ridge. This ridge is not well represented in the model because of the coarse resolution, and the sill in the model is deepened to get a more realistic strength of the overflow. This may influence the representation of the AMOC, however, Kösters et al. (2005) found the effects of an artificially deepened Denmark Strait to be similar to the effects of parameterizing a hydraulically controlled flow over the sill.

The mixing parameterization used in this study must be understood as a first approximation to a better representation of vertical mixing in the ocean. As indicated from the wide range of values for $\alpha$ found in measuring studies, stratification is not the only variable influencing diffusion. It can thus be argued that while essentially unknown, a typical value of $\alpha$ will depend on the general state of the climate system, and thus may have been different during glacial times. One may therefore speculate that the feedback proposed in this study may have contributed to changes in the stability of the $\mathrm{AMOC}$ as inferred from paleoclimate records.

\section{Summary}

Stratification-dependent mixing was implemented into a coupled climate model using $\kappa \sim N^{-\alpha}$, and the sensitivity of the Atlantic MOC to an anomalous freshwater flux was tested for different values of $\alpha$.

Our two main results can be summarized as follows:

1) The "freshwater boosted" regime found by, for example, Nilsson and Walin (2001) and Marzeion and Drange (2006) could not be reproduced in this model. The reason is the buildup of a shallow, warm anomaly in low latitudes that acts as a counterpart to the deeper return flow of the fresh anomaly. As a result, the stratification around pycnocline depth is rather increased than decreased, if only weakly.

2) Two separate regimes could be identified in the response of the overturning to an anomalous freshwater flux: For subcritical values of $\alpha$, the response is a moderate weakening of the overturning. For supercritical values of $\alpha$, a positive feedback between increased stratification and weakened mixing in high latitudes leads to the formation of a stratified barrier layer in the high latitudes, stopping deep convection in the Nordic seas.

Acknowledgments. Author BM was supported under the NOClim 2 project of the Research Council of Norway. Authors AL and JM were supported by the Gary Comer Foundation. The authors thank two anonymous reviewers, whose comments helped to improve the manuscript significantly.

\section{REFERENCES}

Broecker, W. S., and T. Peng, 1982: Tracers in the Sea. Eldigio Press, 690 pp.

Church, J. A., and Coauthors, 2001: Changes in sea level. Climate Change 2001: The Scientific Basis, J. T. Houghton et al., Eds., Cambridge University Press, 640-693.

Clarke, P. U., N. G. Pisias, T. F. Stocker, and A. J. Weaver, 2002: The role of the thermohaline circulation in abrupt climate change. Nature, 415, 863-869.

Fichefet, T., and M. Maqueda, 1997: Sensitivity of a global sea ice model to the treatment of ice thermodynamics and dynamics. J. Geophys. Res., 102, 12 609-12 646.

Ganopolski, A., and S. Rahmstorf, 2001: Rapid changes of glacial climate simulated in a coupled climate model. Nature, 409, 153-158.

Gargett, A. E., and G. Holloway, 1984: Dissipation and diffusion by internal wave breaking. J. Mar. Res., 42, 15-27.

Gnanadesikan, A., 1999: A simple predictive model for the structure of the oceanic pycnocline. Science, 283, 2077-2079.

Gregg, M. C., 1989: Scaling turbulent dissipation in the thermocline. J. Geophys. Res., 94, 9686-9698.

Hasumi, H., and N. Suginohara, 1999: Effects of locally enhanced vertical diffusivity over rough bathymetry on the world ocean circulation. J. Geophys. Res., 104, 23 367-23 374.

Hoffert, M. I., and W. S. Broecker, 1978: Apparent vertical eddy diffusion rates in the pycnocline of the Norwegian Sea as determined from the vertical distribution of tritium. Geophys. Res. Lett., 5, 502-504.

Hofmann, M., and M. Maqueda, 2006: Performance of a secondorder moments advection scheme in an ocean general circulation model. J. Geophys. Res., 111, C05006, doi:10.1029/ 2005JC003279.

Huang, R. X., 1999: Mixing and energetics of the oceanic thermohaline circulation. J. Phys. Oceanogr., 29, 727-746.

Klinger, B. A., and J. Marotzke, 1999: Behavior of doublehemisphere thermohaline flows in a single basin. J. Phys. Oceanogr., 29, 382-399.

Kösters, F., R. H. Käse, A. Schmittner, and P. Herrmann, 2005: The effect of Denmark Strait overflow on the Atlantic me- 
ridional overturning circulation. Geophys. Res. Lett., 32, L04602, doi:10.1029/2004GL022112.

Kuhlbrodt, T., A. Griesel, M. Montoya, A. Levermann, M. Hofmann, and S. Rahmstorf, 2007: On the driving processes of the Atlantic meridional overturning circulation. Rev. Geophys., 45, RG2001, doi:10.1029/2004RG000166.

Large, W. G., J. C. McWilliams, and S. C. Doney, 1994: Oceanic vertical mixing: A review and a model with nonlocal boundary layer parameterization. Rev. Geophys., 32, 363-403.

Levermann, A., and A. Griesel, 2004: Solution of a model for the oceanic pycnocline depth: Scaling of overturning strength and meridional pressure difference. Geophys. Res. Lett., 31, L17302, doi:10.1029/2004GL020678.

Manabe, S., and R. Stouffer, 1995: Simulation of abrupt climate change induced by freshwater input to the North Atlantic Ocean. Nature, 378, 165-167.

Marotzke, J., and J. Scott, 1999: Convective mixing and the thermohaline circulation. J. Phys. Oceanogr., 29, 2962-2970.

— metric thermohaline circulations. J. Phys. Oceanogr., 30, 955970.

Marzeion, B., and H. Drange, 2006: Diapycnal mixing in a conceptual model of the Atlantic Meridional Overturning Circulation. Deep-Sea Res. II, 53, 226-238.

Mignot, J., A. Levermann, and A. Griesel, 2006: A decomposition of the Atlantic meridional overturning circulation into physical components using its sensitivity to vertical diffusivity. $J$. Phys. Oceanogr., 36, 636-650.

Montoya, M., A. Griesel, A. Levermann, J. Mignot, M. Hofmann, A. Ganopolski, and S. Rahmstorf, 2005: The earth system model of intermediate complexity CLIMBER-3 $\alpha$. Part I: Description and performance for present day conditions. Climate Dyn., 25, 237-263.

Munk, W., and C. Wunsch, 1998: Abyssal recipes II: Energetics of tidal and wind mixing. Deep-Sea Res. I, 45, 1977-2010.

Nilsson, J., and G. Walin, 2001: Freshwater forcing as a booster of thermohaline circulation. Tellus, 53A, 629-641.

—, G. Broström, and G. Walin, 2003: The thermohaline circulation and vertical mixing: Does weaker density stratification give stronger overturning? J. Phys. Oceanogr., 33, 27812795.

Oliver, K. I. C., A. J. Watson, and D. P. Stevens, 2005: Can limited ocean mixing buffer rapid climate change? Tellus, 57A, 676690.
Petoukhov, V., A. Ganopolski, V. Brovkin, M. Claussen, A. Eliseev, C. Kubatzki, and S. Rahmstorf, 2000: CLIMBER 2: A climate system model of intermediate complexity. Part I: Model description and performance for present climate. Climate Dyn., 16, 1-17.

Prather, M. J., 1986: Numerical advection by conservation of second-order moments. J. Geophys. Res., 91, 6671-6681.

Rahmstorf, S., 1996: On the freshwater forcing and transport of the Atlantic thermohaline circulation. Climate Dyn., 12, 799 811.

- 2002: Ocean circulation and climate during the past 120,000 years. Nature, 419, 207-214.

- and Coauthors, 2005: Thermohaline circulation hysteresis: A model intercomparison. Geophys. Res. Lett., 32, L23605, doi:10.1029/2005GL023655.

Rehmann, C. R., and T. F. Duda, 2000: Diapycnal diffusivity inferred from scalar microstructure measurements near the New England shelf/slope front. J. Phys. Oceanogr., 30, 13541371.

Rind, D., G. Russell, G. Schmidt, S. Sheth, D. Collins, P. deMenocal, and J. Teller, 2001: Effects of glacial meltwater in the GISS coupled atmosphere-ocean model. 2. A bipolar seesaw in Atlantic deep water production. J. Geophys. Res., 106, $27355-27366$

Sarmiento, J. L., H. W. Feely, W. S. Moore, A. E. Bainbridge, and W. S. Broecker, 1976: The relationship between vertical eddy diffusion and buoyancy gradient in the deep sea. Earth Planet. Sci. Lett., 32, 357-370.

Scott, J. R., J. Marotzke, and P. H. Stone, 1999: Interhemispheric thermohaline circulation in a coupled box model. J. Phys. Oceanogr., 29, 351-365.

Stocker, T. F., and D. G. Wright, 1991: Rapid transitions of the ocean's deep circulation induced by changes in surface water fluxes. Nature, 351, 729-732.

Stouffer, R. J., and Coauthors, 2006: Investigating the causes of the response of the thermohaline circulation to past and future climate changes. J. Climate, 19, 1365-1387.

Vellinga, M., R. A. Wood, and J. M. Gregory, 2002: Processes governing the recovery of a perturbed thermohaline circulation in HadCM3. J. Climate, 15, 764-780.

Zickfeld, K., A. Levermann, M. G. Morgan, T. Kuhlbrodt, S. Rahmstorf, and D. W. Keith, 2007: Present state and future fate of the Atlantic meridional overturning circulation as viewed by experts. Climatic Change, 82, 235-265. 


\section{CORRIGENDUM}

Because of a production error in "The Role of Stratification-Dependent Mixing for the Stability of the Atlantic Overturning in a Global Climate Model," by Ben Marzeion, Anders Levermann, and Juliette Mignot, which appeared in the Journal of Physical Oceanography, Vol. 37, No. 11, 2672-2681, the key to the colored lines that appears in the left panel of Fig. 2 is missing the symbol $\alpha$ in front of the equals signs. The corrected figure is shown below.

The staff of the Journal of Physical Oceanography regrets any inconvenience this error may have caused.
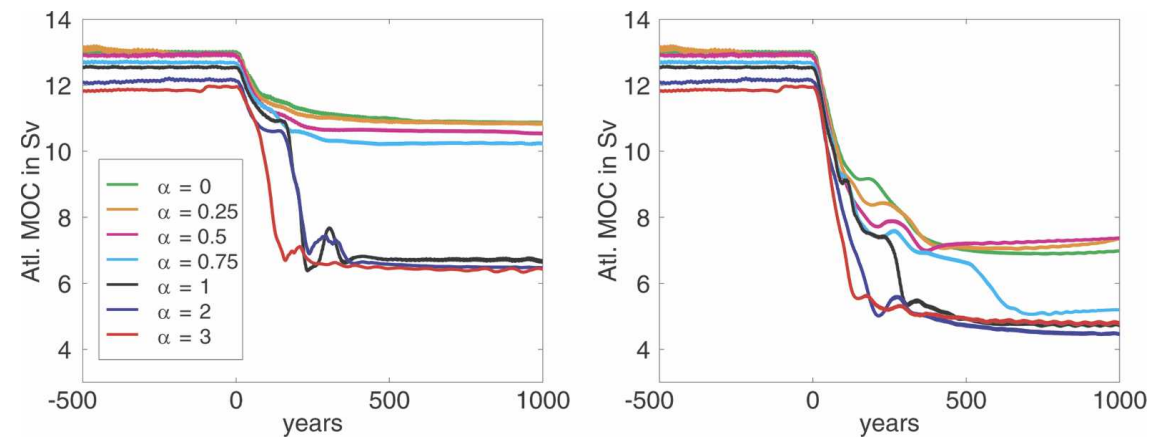

FIG. 2. Time series of the maximum of the Atlantic meridional overturning, filtered with a $20-\mathrm{yr}$ running mean. The freshwater flux starts at $t=0 \mathrm{yr}$. The (left) sflx01 runs and (right) sflx02 runs. 